\title{
A new primal-dual path-following method for convex quadratic programming
}

\author{
MOHAMED ACHACHE \\ Département de Mathématiques, Faculté des Sciences \\ University Ferhat Abbas, Sétif 19000, Algérie \\ E-mail: achache_m@yahoo.fr
}

\begin{abstract}
In this paper, we describe a new primal-dual path-following method to solve a convex quadratic program $(\mathrm{QP})$. The derived algorithm is based on new techniques for finding a new class of search directions similar to the ones developed in a recent paper by Darvay for linear programs. We prove that the short-update algorithm finds an $\varepsilon$-solution of (QP) in a polynomial time.
\end{abstract}

Mathematical subject classification: 90C20, 90C51, 90C60.

Key words: convex quadratic programming, interior point methods, primal-dual path-following methods, convergence of algorithms.

\section{Introduction}

In this paper we consider the following convex quadratic program in its classical standard (QP) form called the primal

$$
\underset{x}{\operatorname{minimize}}\left[c^{T} x+\frac{1}{2} x^{T} Q x\right] \text {, s.t. } A x=b, x \geqslant 0,
$$

and its dual form

$$
\underset{y, x, z}{\operatorname{maximize}}\left[b^{T} y-\frac{1}{2} x^{T} Q x\right], \text { s.t. } A^{T} y+z-Q x=c, z \geqslant 0,
$$

\#636/05. Received: 14/VI/05. Accepted: 26/X/05. 
where $Q$ is a given $(n \times n)$ matrix, $c \in \mathbb{R}^{n}, b \in \mathbb{R}^{m}$ and $A$ is a given $(m \times n)$ matrix.

Recently, some other important formulations have been used to study convex quadratic program such as the (second-order) cone optimization problem (SOCP). They are currently the most often used for quadratic convex programs. See, e.g., [5].

Quadratic programming is a special case of nonlinear programming and has a wide range of applications. A classical method to solve convex (QP) is the Wolfe algorithm which can be considered as a direct extension of the well-known simplex method, in the worst case its complexity is exponential. Therefore, there is a growing interest for developing efficient, robust and polynomial time algorithms to solve the problem. Primal-dual path-following methods are among the most efficient algorithms of interior point methods (IPM) to solve linear, quadratic programming, complementarity problems $(\mathrm{CP})$ and general conic optimization problems (COP). These algorithms are, on one hand, of Newton type and thus lead to an efficient practical implementation and on another hand they have a polynomial time complexity. Recently, many interior point methods have been proposed for solving $(\mathrm{QP})$ problems in its standard format or via (SCOP). These methods are based on new search directions with best theoretical properties such as polynomial time complexity and efficient practical implementations. See, e.g., $[2,4,5,6,7]$. In this paper, we extend a recent approach developed by Darvay [6] for linear programs to $(\mathrm{QP})$ case. In this approach, based on the introduction of a monotonic function $\varphi$ of one real variable. The equations $x_{i} z_{i}=\mu$ in the nonlinear system which defines the central path (see Wright [8]) are replaced by

$$
\varphi\left(\frac{x_{i} z_{i}}{\mu}\right)=\varphi(1) .
$$

Of course, if $\varphi$ is the identical function we recover the classical path-following method. For $\varphi(t)=\sqrt{t}$ we define a new class of search directions and hence a new primal-dual path-following method for approximating the central path. We prove that the related short-update algorithm solves (QP) locally and quadratically and in a polynomial time. We mention also some related and interesting IPM approaches studied recently by Bai et al. [4]. They have also defined a new class of search direction for linear optimization by using the so-called kernel 
function, that is any univariate strictly convex function $k(t)$ that is minimal at $t=1$ and such that $k(1)=0$. However, by defining

$$
k(t)=\int_{1}^{t} \frac{\varphi\left(\xi^{2}\right)-\varphi(1)}{\xi \varphi^{\prime}\left(\xi^{2}\right)} d \xi \quad \text { and for } \quad \varphi(t)=\sqrt{t}, k(t)=(t-1)^{2}
$$

that gives the corresponding kernel function which defines exactly the same search direction and complexity. Nevertheless, for $\varphi(t)=t$ it corresponds to the kernel function $k(t)=\frac{1}{2}\left(t^{2}-1\right)-\log t$ of the logarithmic barrier function, which gives the classical search direction. This new search direction is also analyzed by the authors (Peng, Roos and Terlaky) in a more general context of Self-regular proximities measures for linear and (second order) cone optimization problem. See, e.g., [7].

The paper is organized as follows. In the next section, the problem is presented. Section 3 deals with the new search directions. The description of the algorithm and its convergence analysis are presented in section 4 . Section 5 ends the paper with a conclusion.

Our notation is the classical one. In particular, $\mathbb{R}^{n}$ denotes the space of real $n$-dimensional vectors. Given $u, v \in \mathbb{R}^{n}, u^{T} v$ is their inner product, and $\|u\|$ is the Euclidean norm, whereas $\|u\|_{\infty}$ is the $l_{\infty}$-norm. Given a vector $u$ in $\mathbb{R}^{n}$, $U=\operatorname{diag}(u)$ is the $n \times n$ diagonal matrix with $U_{i i}=u_{i}$ for all $i$.

\section{The statement of the problem}

Through the paper the following assumptions hold.

- Positive semidefinitness (PSD). The matrix $Q$ is symmetric and positive semidefinite.

- Interior point condition (IPC). There exists $\left(x^{0}, y^{0}, z^{0}\right)$ such that $A x^{0}=$ $b, A^{T} y^{0}+z^{0}-Q x^{0}=c, x^{0}>0, z^{0}>0$.

- The full rank condition (FR). The matrix $A$ is of rank $m(m \leqslant n)$. 
The optimal solutions of the primal and the dual are the solutions of nonlinear system of $(2 n+m)$ of equations:

$$
\begin{gathered}
A x=b, \quad x \geqslant 0 \\
A^{T} y+z-Q x=c, z \geqslant 0, \\
x_{i} z_{i}=0, \quad \text { for } i=1, \ldots, n .
\end{gathered}
$$

The first equation of the system (3) represents the primal feasibility, the second one the dual feasibility and the last one the complementarity condition.

The classical path-following method consists in introducing a positive parameter $\mu$. One considers the nonlinear system parameterized by $\mu$

$$
\begin{gathered}
A x=b, \\
A^{T} y+z-Q x=c, z>0, x>0, \\
x_{i} z_{i}=\mu, \text { for } i=1, \ldots, n
\end{gathered}
$$

It is shown, under our assumptions, that there exists one unique solution $(x(\mu), y(\mu), z(\mu))$. The path $\mu \rightarrow(x(\mu), y(\mu), z(\mu))$ is called the central-path (see for instance Wright [8]). It is known that when $\mu \mapsto 0,(x(\mu), y(\mu), z(\mu)$ ) goes to a solution of (3). Applying the Newton's method for the system (4), we develop the classical primal-dual path-following algorithm.

In the next section, we shall present a new method for approximating the central path.

\section{New search directions}

In the proposed method, we replace the $n$ equations $x_{i} y_{i}=\mu$ in (4) by $n$ equivalent equations $\varphi\left(\frac{x_{i} y_{i}}{\mu}\right)=\varphi(1)$ where $\varphi$ is a real valued function on $[0,+\infty)$ and differentiable on $(0,+\infty)$ such that $\varphi$ and $\varphi^{\prime}(t)>0$, for all $t>0$.

Given $\mu>0$ and $(x, y, z)$ such that $A^{T} y+z-Q x=c, A x=b, x>0$ and $z>0$ but not such that $\varphi\left(\frac{x_{i} z_{i}}{\mu}\right)=\varphi(1)$ for all $i$, a new triple $(x+\Delta x, y+$ $\Delta y, z+\Delta z)$ is obtained thanks to the Newton method for solving the nonlinear 
system. One obtains

$$
\begin{gathered}
A \Delta x=0, \\
A^{T} \Delta y+\Delta z-Q \Delta x=0, \\
\frac{1}{\mu} \varphi^{\prime}\left(\frac{x_{i} z_{i}}{\mu}\right)\left(z_{i} \Delta x_{i}+x_{i} \Delta z_{i}\right)=\varphi(1)-\varphi\left(\frac{x_{i} z_{i}}{\mu}\right) \text { for } i=1, \ldots, n .
\end{gathered}
$$

For commodity let us introduce the vectors $v$ and $p$ of $\mathbb{R}^{n}$ defined by

$$
v_{i}=\sqrt{\frac{x_{i} z_{i}}{\mu}} \quad \text { and } \quad p_{i}=\frac{\varphi(1)-\varphi\left(v_{i}^{2}\right)}{v_{i} \varphi\left(v_{i}^{2}\right)} \quad \text { for } \quad i=1, \ldots, n .
$$

Next let us introduce the vectors

$$
d_{x}=X^{-1} V \Delta x, \quad d_{z}=Z^{-1} V \Delta z, \quad d_{y}=\Delta y
$$

and the matrix

$$
\bar{A}=\frac{1}{\mu} A V^{-1} X \quad \text { and } \quad \bar{Q}=\frac{1}{\mu} V^{-1} X Q V^{-1} X
$$

then the system reduces to the system

$$
\bar{A} d_{x}=0, \quad-\bar{A}^{T} d_{y}-d_{z}+\bar{Q} d_{x}=0, \quad d_{x}+d_{z}=p,
$$

which leads to the system

$$
\left(\begin{array}{ll}
(I+\bar{Q}) & -\bar{A}^{T} \\
-\bar{A} & 0
\end{array}\right)\left(\begin{array}{l}
d_{x} \\
d_{y}
\end{array}\right)=\left(\begin{array}{l}
p \\
0
\end{array}\right)
$$

which is non singular since $\bar{Q}$ is positive semidefinite and $\bar{A}$ has a full rank. Then $d_{z}$ is computed by the formula

$$
d_{z}=p-d_{x}
$$

Remark 3.1. If we adopt $\varphi(t)=t$, then we recover the classical primal-dual path-following method

In this paper, we shall consider $\varphi(t)=\sqrt{t}$. Then $\varphi^{\prime}(t)=\frac{1}{2 \sqrt{t}}$. It follows that

$$
p_{i}=2\left(1-\sqrt{\frac{x_{i} z_{i}}{\mu}}\right)=2\left(1-v_{i}\right), \text { for all } i
$$


or

$$
p=2(e-v)
$$

where $e=(1, \ldots, 1)^{T}$ and the system (5) becomes

$$
\begin{gathered}
A \Delta x=0 \\
A^{T} \Delta y+\Delta z-Q \Delta x=0, \\
z_{i} \Delta x_{i}+x_{i} \Delta z_{i}=\mu v_{i} p_{i} \quad \text { for } i=1, \ldots, n .
\end{gathered}
$$

In addition with the notation in (6), we have

$$
x_{i} \Delta z_{i}+z_{i} \Delta x_{i}=\mu v_{i}\left(d_{x_{i}}+d_{z_{i}}\right) \quad \text { for } i=1, \ldots, n,
$$

and

$$
\mu d_{x_{i}} d_{z_{i}}=\Delta x_{i} \Delta z_{i} \text { for } i=1, \ldots, n .
$$

\section{Description of the algorithm and its convergence analysis}

Now we define a proximity measure for the central path as

$$
\delta(X Z e, \mu)=\frac{\|p\|}{2}=\|e-v\| .
$$

In addition, we define the vector $q$ by

$$
q_{i}=d_{x_{i}}-d_{z_{i}} .
$$

Then

$$
d_{x_{i}} d_{z_{i}}=\frac{p_{i}^{2}-q_{i}^{2}}{4}
$$

and

$$
\|q\| \leqslant\|p\| .
$$

This last inequality follows from

$$
\|p\|^{2}=\|q\|^{2}+2 d_{x}^{T} d_{z}
$$

since $d_{x}^{T} d_{z}=d_{x}^{T}\left(\bar{Q} d_{x}-\bar{A}^{T} d_{y}\right)=d_{x}^{T} \bar{Q} d_{x} \geqslant 0$, with $\bar{A} d_{x}=0$ and $\bar{Q}$ is a positive semidefinite matrix. 


\subsection{The algorithm}

Let $\varepsilon>0$ be the given tolerance, $0<\theta<1$ the update parameter (default $\theta=$ $1 /(2 \sqrt{n})$ ), and $0<\tau<1$ the proximity parameter (default $\tau=\frac{1}{2}$ ). Assume that for the triple $\left(x^{0}, y^{0}, z^{0}\right)$ IPC holds and let $\mu^{0}=\frac{\left(x^{0}\right)^{T} z^{0}}{n}$. In addition, we assume that $\delta\left(X^{0} Z^{0} e, \mu\right)<\tau$.

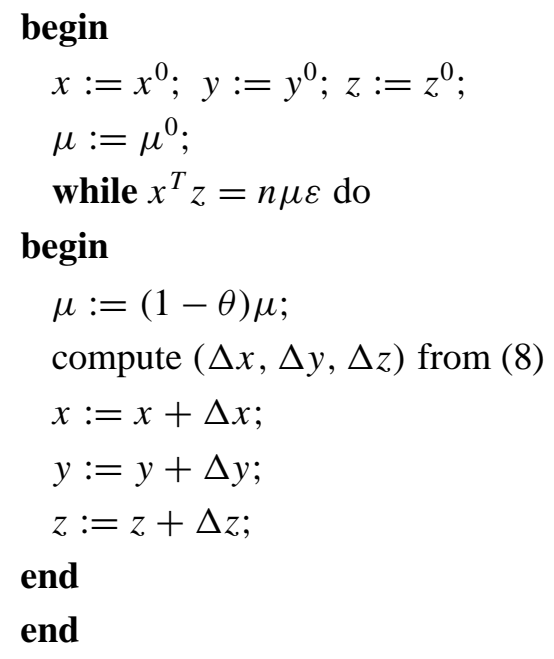

In the next section we prove that the algorithm converges quadratically and locally and in a polynomial time.

\subsection{Complexity analysis}

In the following lemma, we state a condition which ensures the feasibility of the full Newton step. Let $\tilde{x}=x+\Delta x$ and $\tilde{z}=z+\Delta z$ be the new iterate after a full Newton step.

Lemma 4.1. Let $\delta=\delta(X Z e, \mu)<1$. Then the full Newton step is strictly feasible, hence

$$
\tilde{x}>0 \text { and } \tilde{z}>0 .
$$

Proof. For each $0 \leqslant \alpha \leqslant 1$. Let $\tilde{x}(\alpha)=x+\alpha \Delta x$ and $\tilde{z}(\alpha)=z+\alpha \Delta z$. Hence

$$
\tilde{x}_{i}(\alpha) \tilde{z}_{i}(\alpha)=x_{i} z_{i}+\alpha\left(x_{i} \Delta z_{i}+z_{i} \Delta x_{i}\right)+\alpha^{2} \Delta x_{i} \Delta z_{i} \text { for all } i=1, \ldots, n
$$


Now, in view of (9) and (10) we have

$$
\tilde{x}_{i}(\alpha) \tilde{z}_{i}(\alpha)=\mu\left(v_{i}^{2}+\alpha v_{i}\left(d x_{i}+d z_{i}\right)+\alpha^{2} d x_{i} d z_{i}\right) \text { for all } i=1, \ldots, n .
$$

In addition from (7), we have

$$
v_{i}+\frac{p_{i}}{2}=1, \quad \text { for } i=1, \ldots, n .
$$

and thus

$$
v_{i}^{2}+v_{i} p_{i}=1-\frac{p_{i}^{2}}{4} \quad \text { for } i=1, \ldots, n
$$

Thereby

$$
\tilde{x}_{i}(\alpha) \tilde{z}_{i}(\alpha)=\mu\left((1-\alpha) v_{i}^{2}+\alpha\left(v_{i}^{2}+v_{i} p_{i}\right)+\frac{\alpha^{2}}{4}\left(p_{i}^{2}-q_{i}^{2}\right)\right),
$$

and

$$
\tilde{v}_{i}^{2}=(1-\alpha) v_{i}^{2}+\alpha\left(1-(1-\alpha) \frac{p_{i}^{2}}{4}-\alpha \frac{q_{i}^{2}}{4}\right), \quad \text { for } i=1, \ldots, n .
$$

Thus the inequality $\tilde{x}_{i}(\alpha) \tilde{z}_{i}(\alpha)>0$ holds if

$$
\max _{i}\left(\left|(1-\alpha) \frac{p_{i}^{2}}{4}-\alpha \frac{q_{i}^{2}}{4}\right|\right)<1
$$

Using (11) and (12) we get

$$
\begin{aligned}
\max _{i}\left(\left|(1-\alpha) \frac{p_{i}^{2}}{4}-\alpha \frac{q_{i}^{2}}{4}\right|\right) & \leqslant(1-\alpha)\left\|\frac{p}{4}\right\|_{\infty}^{2}+\alpha\left\|\frac{q}{4}\right\|_{\infty}^{2} \\
& \leqslant(1-\alpha) \frac{\|p\|^{2}}{4}+\alpha \frac{\|q\|^{2}}{4} \\
& \leqslant \frac{\|p\|^{2}}{4}=\left(\frac{\|p\|}{2}\right)^{2}=\delta^{2}<1 .
\end{aligned}
$$

Hence, $\tilde{x}_{i}(\alpha) \tilde{z}_{i}(\alpha)>0$ for each $0 \leqslant \alpha \leqslant 1$. Since $\tilde{x}_{i}(\alpha)$ and $\tilde{z}_{i}(\alpha)$ are linear function of $\alpha$, then they do not change sign on the interval $[0,1]$ and for $\alpha=0$ we have $\tilde{x}_{i}(0)>0$ and $\tilde{z}_{i}(0)>0$. This leads to $\tilde{x}_{i}(1)>0$ and $\tilde{z}_{i}(1)>0$ for all $i$.

In the next lemma we state that under the condition $\delta(X Z e, \mu)<1$, the full Newton step is quadratically convergent. 
Lemma 4.2. Let $\tilde{x}=x+\Delta x$ and $\tilde{z}=z+\Delta z$ be the iteration obtained after a full step. Suppose $\delta(X Z e, \mu)<1$. Then

$$
\delta(\tilde{X} \tilde{Z} e, \mu) \leqslant \frac{\delta^{2}}{1+\sqrt{1-\delta^{2}}} .
$$

Thus $\delta(\tilde{X} \tilde{Z} e, \mu)<\delta^{2}$, which means quadratic convergence of the full Newton step.

Proof. Setting in (13) $\alpha=1$, we get

$$
\tilde{v}_{i}^{2}=1-\frac{q_{i}^{2}}{4} \quad \text { for } i=1, \ldots, n
$$

Hence

$$
\min _{i} \tilde{v}_{i}^{2} \geqslant 1-\frac{\|q\|_{\infty}^{2}}{4} \geqslant 1-\frac{\|q\|^{2}}{4} \geqslant 1-\frac{\|p\|^{2}}{4}=1-\delta^{2},
$$

and thereby

$$
\min _{i} \tilde{v}_{i} \geqslant \sqrt{1-\delta^{2}}
$$

On the other hand, we have

$$
\begin{aligned}
\delta^{2}(\tilde{X} \tilde{Z} e, \mu) & =\sum_{i=1}^{n}\left(1-\tilde{v}_{i}\right)^{2} \\
& =\sum_{i=1}^{n}\left(1-\tilde{v}_{i}\right)^{2} \frac{\left(1+\tilde{v}_{i}\right)^{2}}{\left(1+\tilde{v}_{i}\right)^{2}} \\
& =\sum_{i=1}^{n} \frac{\left(1-\tilde{v}_{i}^{2}\right)^{2}}{\left(1+\tilde{v}_{i}\right)^{2}} \\
& \leqslant \frac{1}{\left(1+\min _{i} \tilde{v}_{i}\right)^{2}} \sum_{i=1}^{n}\left(1-\tilde{v}_{i}^{2}\right)^{2}
\end{aligned}
$$

and using (15) we get

$$
\delta^{2}(\tilde{X} \tilde{Z} e, \mu) \leqslant \frac{1}{\left(1+\sqrt{1-\delta^{2}}\right)^{2}} \sum_{i=1}^{n}\left(1-\tilde{v}_{i}^{2}\right)^{2}
$$


Using (11), (12) and (14) we get

$$
\begin{aligned}
\delta^{2}(\tilde{X} \tilde{Z} e, \mu) & \leqslant \frac{\sum_{i=1}^{n} q_{i}^{4}}{4^{2}\left(1+\sqrt{1-\delta^{2}}\right)^{2}} \\
& \leqslant \frac{\left(\sum_{i=1}^{n} q_{i}^{2}\right)^{2}}{4^{2}\left(1+\sqrt{1-\delta^{2}}\right)^{2}}=\frac{1}{\left(1+\sqrt{1-\delta^{2}}\right)^{2}}\left(\frac{\|q\|^{2}}{4}\right)^{2} \\
& \leqslant \frac{1}{\left(1+\sqrt{1-\delta^{2}}\right)^{2}}\left(\frac{\|p\|^{2}}{4}\right)^{2} .
\end{aligned}
$$

Hence

$$
\delta(\tilde{X} \tilde{Z} e, \mu) \leqslant \frac{\delta^{2}}{1+\sqrt{1-\delta^{2}}} .
$$

This proves the Lemma.

The next Lemma gives an upper bound for the duality gap after a full Newton step.

Lemma 4.3. Let $\tilde{x}=x+\Delta x$ and $\tilde{z}=z+\Delta z$. Then the duality gap is

$$
\tilde{x}^{T} \tilde{z}=\mu\left(n-\frac{\|q\|^{2}}{4}\right),
$$

hence

$$
\tilde{x}^{T} \tilde{z} \leq \mu n .
$$

Proof. We have seen from the previous lemmas that

$$
\tilde{v}_{i}^{2}=1-\frac{q_{i}^{2}}{4} \text { for } i=1, \ldots, n .
$$

Hence

$$
\sum_{i=1}^{n} \tilde{v}_{i}^{2}=\sum_{i=1}^{n}\left(1-\frac{q_{i}^{2}}{4}\right)=n-\frac{\|q\|^{2}}{4}
$$

but since

$$
\tilde{x}^{T} \tilde{z}=\mu \sum_{i=1}^{n} \tilde{v}_{i}^{2}
$$


it follows that

$$
\tilde{x}^{T} \tilde{z}=\mu\left(n-\frac{\|q\|^{2}}{4}\right) .
$$

This completes the proof.

The next Lemma discusses the influence on the proximity measure of the Newton process followed by a step along the central path.

Lemma 4.4. Let $\delta=\delta(X Z e, \mu)<1$ and $\mu_{+}=(1-\theta) \mu$, where $0<\theta<1$.

Then

$$
\delta\left(\tilde{X} \tilde{Z} e, \mu_{+}\right) \leq \frac{\theta \sqrt{n}+\delta}{1-\theta+\sqrt{(1-\theta)(1-\delta)}} .
$$

Furthermore, if $\delta<1 / 2, \theta=1 /(2 \sqrt{n})$ and $n \geqslant 4$, then we get

$$
\delta\left(\tilde{X} \tilde{Z} e, \mu_{+}\right) \leqslant \frac{1}{2} .
$$

Proof. We have

$$
\begin{aligned}
\delta^{2}\left(\tilde{X} \tilde{Z} e, \mu_{+}\right) & =\left\|e-\sqrt{\frac{\tilde{x} \tilde{y}}{\mu_{+}}}\right\|^{2} \\
& =\frac{1}{1-\theta}\|\sqrt{1-\theta} e-\tilde{v}\|^{2} \\
& =\frac{1}{1-\theta} \sum_{i=1}^{n}\left(\sqrt{1-\theta}-\tilde{v}_{i}\right)^{2} \\
& =\frac{1}{1-\theta} \sum_{i=1}^{n}\left(\sqrt{1-\theta}-\tilde{v}_{i}\right)^{2} \frac{\left(\sqrt{1-\theta}+\tilde{v}_{i}\right)^{2}}{\left(\sqrt{1-\theta}+\tilde{v}_{i}\right)^{2}} \\
& =\frac{1}{1-\theta} \sum_{i=1}^{n} \frac{\left((1-\theta)-\tilde{v}_{i}^{2}\right)^{2}}{\left(\sqrt{1-\theta}+\tilde{v}_{i}\right)^{2}} \\
& \leqslant \frac{1}{1-\theta} \frac{1}{\left(\sqrt{1-\theta}+\min _{i} \tilde{v}_{i}\right)^{2}} \sum_{i=1}^{n}\left((1-\theta)-\tilde{v}_{i}^{2}\right)^{2}
\end{aligned}
$$


Hence by using (13) and (14) we get

$$
\delta\left(\tilde{X} \tilde{Z} e, \mu_{+}\right) \leqslant \frac{1}{\sqrt{1-\theta}} \frac{1}{\left(\sqrt{(1-\theta)}+\sqrt{\left(1-\delta^{2}\right)}\right)} \sqrt{\sum_{i=1}^{n}\left(-\theta+\frac{q_{i}^{2}}{4}\right)^{2}}
$$

Now, in view of (10) and (11) we deduce that

$$
\begin{aligned}
\delta\left(\tilde{X} \tilde{Z} e, \mu_{+}\right) & \leqslant \frac{1}{1-\theta+\sqrt{(1-\theta)\left(1-\delta^{2}\right)}}\left[\theta \sqrt{n}+\left(\frac{\|q\|}{2}\right)\right] \\
& \leqslant \frac{1}{1-\theta+\sqrt{(1-\theta)\left(1-\delta^{2}\right)}}\left[\theta \sqrt{n}+\left(\frac{\|p\|}{2}\right)\right] .
\end{aligned}
$$

Finally, we get

$$
\delta\left(\tilde{X} \tilde{Z} e, \mu_{+}\right) \leqslant \frac{1}{1-\theta+\sqrt{(1-\theta)\left(1-\delta^{2}\right)}}[\theta \sqrt{n}+\delta] .
$$

This completes the proof.

It results from Lemma 4.4 that for the default $\theta=1 /(2 \sqrt{n})$, the $(x, z)>0$ and $\delta(X Z e, \mu)<1 / 2$ are maintained during the algorithm. Hence the algorithm is well defined.

In the next Lemma we compute an upper bound for the total number of iterations produced by the algorithm.

Lemma 4.5. Assume that $x^{0}$ and $z^{0}$ are strictly feasible, $\mu^{0}=\frac{\left(x^{0}\right)^{T} z^{0}}{n}$ and $\delta\left(X^{0} Z^{0} e, \mu\right)<\frac{1}{2}$. Moreover, let $x^{k}$ and $z^{k}$ be the vectors obtained after $k$ iterations. Then the inequality $\left(x^{k}\right)^{T} z^{k} \leqslant \varepsilon$ is satisfied for

$$
k \geqslant\left[\frac{1}{\theta} \log \frac{\left(x^{0}\right)^{T} z^{0}}{\varepsilon}\right]
$$

Proof. We have after $k$ iterations that $\mu^{k}=(1-\theta)^{k} \mu^{0}$. Using Lemma 4.3, it follows that $\left(x^{k}\right)^{T} z^{k} \leqslant n \mu^{k}=(1-\theta)^{k}\left(x^{0}\right)^{T} z^{0}$. Hence the inequality $\left(x^{k}\right)^{T} z^{k} \leqslant \varepsilon$ holds if $(1-\theta)^{k}\left(x^{0}\right)^{T} z^{0} \leqslant \varepsilon$. By taking logarithms of both sides, we obtain

$$
k \log (1-\theta)+\log \left(x^{0}\right)^{T} z^{0} \leqslant \log \varepsilon .
$$


The estimate for the log function

$$
-\log (1-\theta) \geqslant \theta \text { for all } 0 \leqslant \theta<1 .
$$

Therefore the inequality $\left(x^{k}\right)^{T} z^{k} \leqslant \varepsilon$ is fulfilled if $k \geqslant \frac{1}{\theta} \log \frac{\left(x^{0}\right)^{T} z^{0}}{\varepsilon}$.

For the default $\theta=1 /(2 \sqrt{n})$, we obtain the following theorem.

Theorem 4.6. Suppose that the pair $\left(x^{0}, z^{0}\right)$ is strictly feasible and let $\mu^{0}=$ $\left(x^{0}\right)^{T} z^{0} / n$. If $\theta=1 /(2 \sqrt{n})$, then the algorithm 4.1 requires at most

$$
\left[2 \sqrt{n} \log \frac{\left(x^{0}\right)^{T} z^{0}}{\varepsilon}\right]
$$

iterations. For the resulting vectors we have $\left(x^{k}\right)^{T} z^{k} \leqslant \varepsilon$.

\section{Conclusion}

In this paper, we have described a new primal-dual path-following method to solve convex quadratic programs. We have proved that the short-update algorithm can find an $\varepsilon$-solution for (QP) in

$$
2 \sqrt{n} \log \frac{\left(x^{0}\right)^{T} z^{0}}{\varepsilon}
$$

iterations, where $x^{0}$ and $z^{0}$ are the initial positive starting points. The initialization of the algorithm can be achieved as in linear optimization by using self-dual embedding techniques. Hence, if $x^{0}=y^{0}=e$, we get the best well-known short step complexity

$$
2 \sqrt{n} \log \left(\frac{n}{\varepsilon}\right) .
$$

\section{REFERENCES}

[1] L. Adler and R.D.C. Monteiro, Interior path following primal-dual algorithms, Part II: Convex quadratic programming. Mathematical Programming, 44 (1989), 43-66.

[2] F. Alizadeh and D. Goldfarb, Second-order cone programming. Mathematical programming, 95 (2003), 3-15.

[3] K.M. Anstreicher, D. den hertog, C. Roos and T. Terlaky, A long step barrier method for convex quadratic programming. Delft University of technology. Report, (1990), 90-53. 
[4] Y.Q. Bai, M. El ghami and C. Roos, A new efficient large-update primal-dual interior-point method based on a finite barrier. SIAM Journal on Optimization, 13(3) (2003), 766-782.

[5] A. Ben-Tal and A. Nemirovski, Lectures on Modern convex Optimization. Analysis, Algorithms and engineering Applications, volume 2 of MPS-SIAM Series on Optimization. SIAM, Philadelphia, USA (2001).

[6] Zs. Darvay, A new algorithm for solving self-dual linear programming problems. Studia Universitatis Babes-Bolyai, Series Informatica, 47(1) (2002), 15-26.

[7] J. Peng, C. Roos and T. Terlaky, Primal-dual interior point methods for second-order conic optimization based on self-regular proximities. SIAM J. Optimization, 13(1) (2002), 179-203.

[8] S.J. Wright. Primal-Dual Interior Point Methods. SIAM, Philadelphia, (1997), USA. 